\title{
Fracton pairing mechanism for "strange" superconductors: Self-assembling organic polymers and copper-oxide compounds
}

\author{
Alexander V. Milovanov* and Jens J. Rasmussen \\ Optics and Fluid Dynamics Department, Risoe National Laboratory, DK-4000 Roskilde, Denmark \\ * permanent address: Space Research Institute, Profsoyuznaya street, 84/32, 117997 Moscow, Russia
}

Self-assembling organic polymers and copper-oxide compounds are two classes of "strange" superconductors, whose challenging behavior does not comply with the traditional picture of Bardeen, Cooper, and Schrieffer (BCS) superconductivity in regular crystals. In this paper, we propose a theoretical model that accounts for the strange superconducting properties of either class of the materials. These properties are considered as interconnected manifestations of the same phenomenon: We argue that superconductivity occurs in the both cases because the charge carriers (i.e., electrons or holes) exchange fracton excitations, quantum oscillations of fractal lattices that mimic the complex microscopic organization of the strange superconductors. For the copper oxides, the superconducting transition temperature $T_{c}$ as predicted by the fracton mechanism is of the order of $\sim 150$ $\mathrm{K}$. We suggest that the marginal ingredient of the high-temperature superconducting phase is provided by fracton coupled holes that condensate in the conducting copper-oxygen planes owing to the intrinsic field-effect-transistor configuration of the cuprate compounds. For the gate-induced superconducting phase in the electron-doped polymers, we simultaneously find a rather modest transition temperature of $\sim(2-3) \mathrm{K}$ owing to the limitations imposed by the electron tunneling processes on a fractal geometry. We speculate that hole-type superconductivity observes larger onset temperatures when compared to its electron-type counterpart. This promises an intriguing possibility of the high-temperature superconducting states in hole-doped complex materials. The theoretical methods applied in our study bring together the so-called strange (or fractional) dynamics and the unconventional, topological description of the complex fractal sets underlying the fracton spectrum. A generalized kinetic equation containing integer time and fractional real-space derivatives is found for the fracton excitations in the harmonic approximation. The fracton superconductivity mechanism is further discussed in connection with experimental observations. An important prediction of the present study is universality of ac conduction of the strange materials above the superconducting transition temperature $T_{c}$.

61.43.-j, 63.22.+m, 74.10.+v, 72.80.Le, 74.20.Mn 


\section{INTRODUCTION}

The physics of two-dimensional electron gases has been influential in condensed-matter research since the discovery of highly correlated liquid states, such as electron liquids with fractionally charged excitations, ${ }^{1}$ and hightemperature superconducting fluid phases in copperoxide compounds. ${ }^{2}$ The trademark of copper oxides is challenging superconducting transition temperature ${ }^{2}$ (up to $T_{c} \sim 160 \mathrm{~K}$ ); this somehow involves the specific microscopic organization of the cuprate superconductors composed of the alternating conducting-insulating planes.

The fundamental properties of correlated electrons have received attention with the observation of quantum Hall effect in polymeric materials (such as pentracene) ${ }^{3}$ and superconducting behavior in molecular crystals ${ }^{4}$ and organic polymer films. ${ }^{5}$ The discovery of superconducting organic polymers ${ }^{5}$ addressed the correlations between the current-carrying electrons in presence of the multiscale structural disorder associated with the complex microscopic texture of the polymer system. ${ }^{6}$

Organic polymer films ${ }^{5}$ were made to superconduct through charge injection in a field-effect-transistor geometry. In field-effect transistors, a voltage applied to the gate electrode controls the charge flow along the surface of the active (conducting) layer. The active material in the experiment in Ref. ${ }^{5}$ was solution-cast regioregular poly(3-hexylthiophene) (P3HT) deposited on an insulating substrate. A superconducting flow was observed below $T_{c} \sim 2.35 \mathrm{~K}$ for the high gate-induced carrier concentration of about one charge per 12 thiophene rings. The superconducting behavior in P3HT was related to the self-assembly properties of the polymer, which forms thin regular films owing to self-organization. ${ }^{5,6}$

Remark that the gate-induced charge doping introduces no additional disorder into the material, enabling a direct observation of the superconducting transition as a function of temperature and charge carrier concentration. ${ }^{5,6}$ Conversely, chemical doping (i.e., injection of suitable impurities such as chlorine or alkali metals, leading to a surplus or deficit of free electrons) inevitably increases the disorder. ${ }^{5}$ This changes the properties of the conducting system and can suppress the inherent self-organization mechanisms that make organic polymers superconduct. With increasing concentration of the impurities, the superconducting transition temperature $T_{c}$ shifts to lower values, while the transition region becomes generally broader. ${ }^{5}$ Chemical doping has thus defied attempts to turn polymers into superconductors. ${ }^{5,7}$ Until the field-effect experiments in Ref., ${ }^{5}$ the only polymeric material exhibiting superconductivity was a crystalline inorganic polymer polysulfur nitride $(\mathrm{SN})_{X}$ having a transition temperature of $\sim 0.26 \mathrm{~K} .^{8}$

The key issue on the superconducting organic polymers is the comprehension of the electron pairing mechanism. The main problem is that electronic properties of the polymers are dominated by the intrinsic spatial disorder which supports charge localization at low temperatures. ${ }^{6}$ (The effect of the disorder can be provided, for instance, by a multiscale braiding of the polymer molecules.) $\mathrm{Su}-$ perconducting transition in organic polymers have been discussed $^{6}$ in connection with both the conventional picture of Bardeen, Cooper, and Schrieffer (BCS) superconductivity relying on the electron-phonon interactions in regular crystals, ${ }^{9}$ and Little's exciton mechanism, in which phonons are replaced by other electrons. ${ }^{10}$ An alternative approach could be based on a spin-triplet, even-parity superconductivity for disordered, interacting electron systems. ${ }^{11}$ While strong arguments in favour of the traditional explanation in terms of phonon-mediated superconductivity have been proposed, ${ }^{5,6}$ the formation of the Cooper pairs on the disordered polymer patterns (revealing no regular monocrystalline configuration, contrary to the BCS scenario) remains so far generally unclear.

In this paper, we propose a feasible electron pairing mechanism for the "strange" materials like organic polymer P3HT that may observe superconducting behavior in spite of the intrinsic structural disorder. We suggest that electron-electron interactions in such materials are mediated by fractons, quantum oscillations of the multiscale conducting arrays organized in a fractal lattice. The fracton pairing mechanism thereby profits from the complex geometry of the effective conducting set.

It is instructive to emphasize that fracton-mediated interactions between the current-carrying particles could be characteristic of the disordered solids as a whole, rather than specifically of the polymeric materials. Selfassembling organic polymers are considered in our study as a suitable guide to the "strange" superconducting properties of complex systems, while the fracton mechanism is not attributed only to the polymers.

We assume, as a general condition, that the material posseses complex microscopic organization that supports a well-defined branch of fracton excitations. We argue that the fracton modes may then effectively confine the current-carrying electrons in couples below a certain temperature, thus leading to the occurrence of the marginal superconducting fluid state. We demonstrate, furthermore, that replacing electrons with "holes" in the fracton scenario leads to the intriguing hints regarding the nature of the high-temperature superconductivity in copper oxide materials. The fracton approach may thereby indicate that the superconducting properties of organic polymers and of copper oxides are both parts of the same problem.

The paper is organized as follows. The basic assumptions of our study are formulated in Sec. II. A short summary on fracton dynamics is condensed to Sec. III. The fundamental topological properties of percolating fractal lattices giving rise to a well-defined fracton spectrum, are analyzed in Secs. IV $-\mathrm{V}$. The fracton wave function is derived in Sec. VI from a fractional kinetic equation in the harmonic approximation. Based on the results obtained, the superconducting transition in self- 
assembling electron-doped organic polymers is discussed in Secs. VII-VIII. A case for high-temperature superconductivity is analuzed in Sec. IX; both hole-doped complex systems and copper-oxide compounds are considered. Implications of the fracton pairing mechanism on ac conduction properties of the strange superconductors above the marginal transition temperature $T_{c}$ are addressed in Sec. X. The principal conclusions are given in Sec. XI.

\section{FRACTAL GEOMETRY OF PERCOLATION}

We start with a suitable geometric description of the disordered molecular (polymeric) structures. Our basic assumptions are, 1) self-organization of organic polymers results in development of a multiscale conducting network of molecules, 2) the self-organized conducting networks form percolating sets, and 3) these sets are critical, i.e., at the threshold of percolation. A percolating network is defined as an infinite connected web-like configuration embedded in an Euclidean space $E^{d}$, where $d$ is ambient (integer) dimension; in what follows, $d \geq 2$. The term "percolating" is synonymous with "infinite connected" and guarantees that the molecular network conducts on the macroscopic scales. The threshold character means that the percolating web, although infinite in size, is minimally developed in $E^{d}$, i.e., observes a sort of a structural extremum. More precisely, assume the effective conducting web is composed of multiscale conducting links whose concentration in the space $E^{d}$ is quantified by the parameter $0 \leq q \leq 1 .^{12}$ For $q \rightarrow 0$, no connected structure can exist as the web lacks conducting elements on all scales; conversely, for $q \rightarrow 1$, the multiscale conducting links densely fill the ambient space. As $q$ continuously grows from 0 to 1 , an infinite connected structure (which occupies only a fraction of the embedding space $\left.E^{d}\right)$ first appears for $q=q_{c}$, where $0<q_{c}<1$ is some critical concentration of the links. (Roughly speaking, $q_{c}$ is "of the order of one half".) The value $q=q_{c}$ can be identified with the percolation threshold in the system, corresponding to a minimal (critical) network which conducts on large scales. As $q$ approaches $q_{c}$, the percolation correlation (i.e., pair connectedness) length diverges as $\xi \sim\left|q-q_{c}\right|^{-\nu}$, where $\nu$ is the percolation length exponent depending solely on the embedding dimension $d \geq 2$. For $q>q_{c}$, the probability to belong to the infinite connected network behaves as $Q_{\infty}(q) \propto\left(q-q_{c}\right)^{\beta} \propto \xi^{-\beta / \nu}$, while dc conductivity goes to zero as $\Sigma(0) \propto\left(q-q_{c}\right)^{\mu} \propto \xi^{-\mu / \nu}$. Along with the exponent $\nu$, the percolation indices $\beta$ and $\mu$ completely characterize the structural properties of the effective conducting set for $q \rightarrow+q_{c}$. Both $Q_{\infty}(q)$ and $\Sigma(0)$ vanish for $q<q_{c}$.

The microscopic conducting mechanism operating in the polymeric materials such as P3HT is supported by the conjugated structure of alternating single and double bonds linking the carbon atoms in their backbone. ${ }^{6}$
This bonding structure allows one electron from each carbon atom to move along the molecule and become conducting. ${ }^{6,13}$ The multiscale braiding of the polymer molecules gives rise to a complex spatial distribution of the conducting links, modeled in our study by a percolating web-like pattern.

A remarkable property of percolating networks at criticality (i.e., near the marginal threshold $q=q_{c}$ ) is their self-similar (fractal) geometry. ${ }^{12,14-16}$ In a physical system, the fractal geometry approximation holds in a wide but finite range of spatial scales $\chi$, which vary between the microscopic ("lattice") distance $\chi_{\min } \sim a$ and the percolation correlation length $\chi_{\max } \sim \xi \gg a$. Given arbitrary length scale $a \lesssim \chi \lesssim \xi$, one finds the number of the links that belong to the percolating network within the volume $\chi^{d} \subset E^{d}$ to be $N_{\chi} \propto \chi^{d_{f}}$. The quantity $d_{f}$ is the generalized (Hausdorff) dimension of the network which is not larger than $d$, i.e., $d_{f} \leq d .{ }^{14,17}$ Specifically, $d_{f}=d-\beta / \nu$ for $a \lesssim \chi \lesssim \xi .^{12}$ Note that $d_{f} \geq 1$ for fractal networks admitting connected pathes to infinity.

The value of the Hausdorff dimension $d_{f}$ is in one-toone correspondence with the topological entropy of the fractal network, $S_{f}:^{18}$

$$
S_{f}=1-1 / d_{f}
$$

The topological entropy $S_{f}$ measures the structural disorder in complex systems. ${ }^{19}$ For well-ordered conducting polymer chains without braiding, $d_{f} \rightarrow 1$ and $S_{f} \rightarrow 0$. Such conditions could be realized at low absolute temperatures $T \rightarrow 0$. For the multiscale path-connected braided structures, $d_{f}>1$ and $S_{f}>0$. Similar to the conventional, thermodynamic entropy, the topological entropy $S_{f}$ is a non-decreasing function of $T$, i.e., $\partial S_{f} / \partial T \geq 0$. From Eq. (1) one obtains

$$
\partial d_{f} / \partial T \geq 0
$$

At length scales $\chi$ exceeding the correlation length $\xi$, the fractal geometry of percolation crosses over to a statistically homogeneous distribution of the conducting links. This means $N_{\chi} \propto \chi^{d}$ for $\chi \gtrsim \xi$. At the short length scales $\chi \lesssim a$, the material may reveal regular crystalline structure; in polymers like P3HT, this structure can be associated with ordered nanocrystals embedded in a disordered matrix. ${ }^{6}$ The typical size of the nanocrystals is, $a \sim 10^{-6} \mathrm{~cm} .{ }^{5}$ Note that the ratio $\xi / a$ in real fractal materials is at most $\sim 10^{3}$ due to the limitations imposed by thermal vibrations and by gravitational stability. ${ }^{20}$

The formation of fractal conducting networks in the range of scales $a \lesssim \chi \lesssim \xi$ may be explained by selforganized criticality (SOC), a phenomenon which often underlies the development of fractal structures in complex nonlinear dynamical systems. ${ }^{21}$ Here, the concept of SOC is understood as a synonymous with "selforganization to a state of critical percolation." ${ }^{18,22}$ The inherent dynamics of the ideal SOC state is manifested in the low frequency $f^{-1}$ fluctuation spectrum ${ }^{21}$ customarily referred to as flicker noise. The feasible existence 
of the anomalously large flicker noise in resistance fluctuations for disordered solid materials was addressed in Ref. $^{23}$

\section{FRACTON DYNAMICS}

At the quantum level, the dynamics of a percolating fractal network can be described in terms of elementary (quasi)acoustic excitations termed fractons. ${ }^{24}$ Fractons are considered as quantum oscillations of the local disorder in the system. In the harmonic approximation, fractons are well defined quantum states with infinite inelastic lifetimes. ${ }^{25}$ Statistical properties of fractons were reviewed by Nakayama et al. ${ }^{16}$ in connection with the topological characteristics of fractal sets. A direct experimental observation of fracton modes in real complex materials was reported in Ref. ${ }^{20}$

Fracton excitations of the critical percolating networks form a well-defined (quasi)acoustic branch representing vibrations of multiscale fractal blobs. ${ }^{25}$ In fractal geometries, such vibrations acquire the role of ordinary phonons propagating through periodic crystal lattices. (In polymeric materials like P3HT, the "blobs" may be identified with the disordered polycrystalline matrices.) Note that phonons having wavelengths shorter than the percolation correlation length $\xi$ are strongly scattered by the structural inhomogeneitites associated with the fractal geometry of the underlying pattern. Hence phonons cannot be eigen vibrational modes of a fractal. Conversely, fractons are localized states, in the Anderson sense, ${ }^{26}$ with a localization length larger than the characteristic phonon scattering length. ${ }^{20,25}$

The defining feature of the fracton branch is the nonlinear relationship ${ }^{16,24,25}$

$$
\omega \propto q^{\sigma}
$$

between angular frequency $\omega$ and wave vector $q=2 \pi / \lambda$. Here, $\lambda$ is the fracton wavelength, which lies between the characteristic fractal cutoff scales $\chi_{\min } \sim a$ and $\chi_{\max } \sim$ $\xi$, i.e., $a \lesssim \lambda \lesssim \xi$. The power exponent $\sigma$ in the dispersion relation $(3)$ equals

$$
\sigma=(2+\theta) / 2
$$

where $\theta$ is the index of connectivity of the fractal. The index of connectivity determines the microscopic composition of the fractal object through the marginal links between the constituent elements. ${ }^{27}$ The value of $\theta$ does not depend on the way the fractal is folded in the embedding Euclidean space $E^{d}$ (as opposed to the Hausdorff dimension $d_{f}$ which relies on the embedding properties of the fractal). Contrary to the Hausdorff dimension $d_{f}$, the index of connectivity $\theta$ is a topological invariant of the fractal structure, i.e., remains unchanged under the homeomorphic deformations that preserve the property of self-similarity. ${ }^{28}$ The index of connectivity $\theta$ can be expressed in terms of the percolation exponents $\nu, \beta$, and $\mu$ via $\theta=(\mu-\beta) / \nu{ }^{12}$ In Euclidean geometries, $\theta \equiv 0$.

The fracton phase velocity, $c_{\sigma}$, scales with wave vector $q$ as $c_{\sigma}(q) \sim \omega / q \propto q^{\theta / 2}$. For $q \xi / 2 \pi \lesssim 1$, the fracton branch crosses over to the long-wave phonons, whose phase velocity, $c$, is a constant function of $q$, i.e., $c \sim \omega / q=$ const $(q) .{ }^{16,25}$ Note that phonons are characterized by the linear dispersion relation $\omega \propto q$, which follows from Eq. (3) in the Euclidean geometry limit $\theta \rightarrow 0$. In the fractal domain, we have, consequently,

$$
c_{\sigma}(q) \sim c \times(q \xi / 2 \pi)^{\theta / 2}
$$

where the fracton wave vector $q$ varies from $q_{\text {min }} \sim 2 \pi / \xi$ to $q_{\max } \sim 2 \pi / a$. Considering Eq. (5), one concludes that the fracton phase velocity cannot exceed the maximum of

$$
c_{\sigma \max } \sim c \times(\xi / a)^{\theta / 2},
$$

corresponding to the shortest-wave fractons with $q \sim$ $q_{\max }$. Accordingly, the fracton frequecies range through

$$
\omega_{\min } \lesssim \omega \lesssim \omega_{\min } \times(\xi / a)^{\sigma}
$$

where $\omega_{\min } \sim 2 \pi c / \xi$ is the long-wave phonon crossover scale.

The fracton density of states (DOS) behaves with frequency $\omega$ as ${ }^{16,24,25}$

$$
\mathcal{D}_{\mathrm{fr}}(\omega) \propto \omega^{d_{s}-1}
$$

where $d_{s}=2 d_{f} /(2+\theta)=d_{f} / \sigma$ is the so-called spectral fractal dimension ${ }^{24}$ (to be distinguished from the Hausdorff dimension of the fractal, $d_{f}$ ). The value of $d_{s}$ determines the effective (fractional) number of degrees of freedom on a fractal geometry. ${ }^{29}$ In the limit $d_{s} \rightarrow d$, expression (8) crosses over to the phonon $\operatorname{DOS}^{30}$

$$
\mathcal{D}_{\mathrm{ph}}(\omega) \propto \omega^{d-1}
$$

\section{THE PERCOLATION CONSTANT}

Near the threshold of percolation, $q=q_{c}$, the value of the spectral dimension $d_{s}$ can be obtained from topological arguments. ${ }^{31}$ In fact, let $\partial \mathcal{N}_{c}$ and $\mathcal{N}_{c}$ denote the critical and fat percolating networks, respectively. The fat percolating network is defined as the set of points which can be path connected ${ }^{32}$ to $\partial \mathcal{N}_{c}$ for all $q<q_{c}$. Obviously, $\mathcal{N}_{c}$ is an open set, ${ }^{33}$ and $\partial \mathcal{N}_{c}$ is the boundary ${ }^{33}$ of $\mathcal{N}_{c}$. (This motivates the commonly used sign " $\partial$ " in the notation $\partial \mathcal{N}_{c}$.) We also define the closed set $^{33}$ $\overline{\mathcal{N}}_{c}=\mathcal{N}_{c} \bigcup \partial \mathcal{N}_{c}$.

It can be proven ${ }^{31}$ that $\partial \mathcal{N}_{c}$ and $\mathcal{N}_{c}$ are topologically equivalent with the fractional $\left(d_{s}-1\right)$-dimensional sphere $S^{d_{s}-1}$ and the fractional $d_{s}$-dimensional open disk 
$D^{d_{s}}$, respectively, where $S^{d_{s}-1}=\partial D^{d_{s}}$ is the boundary of $D^{d_{s}}$. The introduction of the fractional manifolds ${ }^{31}$ $S^{d_{s}-1}$ and $D^{d_{s}}$ extends the notion of the smooth integerdimensional manifolds ${ }^{32,34} S^{d-1}$ and $D^{d}$ to arbitrary real dimensionalities $d_{s}<d$. Note that the fractional sphere $S^{d_{s}-1}$ is based on the solid angle

$$
\Omega_{d_{s}}=d_{s} \frac{\pi^{d_{s} / 2}}{\Gamma\left(d_{s} / 2+1\right)}
$$

where $\Gamma$ is the Euler gamma-function. From Eq. (10) one recovers the familiar results $\Omega_{2}=2 \pi$ for the standard circle $S^{1}$, and $\Omega_{3}=4 \pi$ for the standard two-dimensional sphere $S^{2}$. The topological equivalence relation can be given by a diffeomorhism $\left\{\phi: \partial \mathcal{N}_{c} \rightarrow S^{d_{s}-1}, \mathcal{N}_{c} \rightarrow\right.$ $\left.D^{d_{s}}\right\}$. (Here, the diffeomorphic relation $\phi$ implies fractional extension ${ }^{31}$ of the Jacobian ${ }^{34}$.)

As is shown in Ref. ${ }^{31}$, the closed fractional manifold $\bar{D}^{d_{s}}=D^{d_{s}} \cup S^{d_{s}-1}$ admits everywhere a dense covering by a fractal curve $\gamma^{*}$ which has no points of selfcrossings at the critical threshold $q=q_{c}$. The absence of points of self-crossings minimizes the topology of the network for $q=q_{c}$ and thereby supports the conditions of the critical percolation. (For $q>q_{c}$, the corresponding manifolds contain "too many points" to be densely covered by a curve without self-crossings. The minimal closed percolating structure $\overline{\mathcal{N}}_{c} \subset E^{d}$ therefore contains "the same number of points" as a fractal curve without self-crossings, folded in $E^{d}$. This serves as the defining feature of the critical percolation. ${ }^{31}$ ) Because $\gamma^{*}$ is dense everywhere in $\bar{D}^{d_{s}}$, it must be based on exactly the same solid angle $\Omega\left(\gamma^{*}\right)=\Omega_{d_{s}}$ as the fractional sphere $S^{d_{s}-1}=\partial D^{d_{s}}$ [see Eq. (10)]. On the other hand, a curve $\gamma^{*}$ cannot be principally based on a solid angle $\Omega\left(\gamma^{*}\right)$ smaller than $\Omega_{2} / 2=\pi$ in $d \geq 2$ embedding dimensions, i.e., $\Omega\left(\gamma^{*}\right) \geq \pi$ for $d \geq 2$. The criticality condition $q=q_{c}$ minimizes the solid angle $\Omega_{d_{s}}$, i.e., $\Omega\left(\gamma^{*}\right)=\Omega_{d_{s}}=\pi$ at the percolation threshold. ${ }^{31}$ The solution to this transcendental algebraic equation is the fundamental topological parameter $\mathcal{C}$ (termed "the percolation constant" in Ref. ${ }^{28}$ ) satisfying, by definition, the identity ${ }^{31}$

$$
\mathcal{C} \frac{\pi^{\mathcal{C} / 2}}{\Gamma(\mathcal{C} / 2+1)}=\pi
$$

Numerically, $\mathcal{C} \approx 1.327 .{ }^{31}$ Hence, the spectral fractal dimension

$$
d_{s} \equiv 2 d_{f} /(2+\theta)=\mathcal{C} \approx 1.327
$$

at $q=q_{c}$. Based on the Whitney embedding theorem, ${ }^{35}$ one may demonstrate ${ }^{31}$ that Eq. (12) holds for embedding dimensions $d$ between 2 and 5, i.e., $2 \leq d \leq 5$. Note that the value of $d_{s}$ at $q=q_{c}$ is given by the mean-field theory $^{36}$ for all $d \geq 6: d_{s}=4 / 3$. Below the critical embedding dimension of $d=6$, the mean-field percolation is invalidated; ${ }^{16,36}$ this appears in the deviation of the percolation constant $\mathcal{C}$ from $4 / 3$ for $2 \leq d \leq 5$.
The fact that $\mathcal{C}$ is actually smaller than $4 / 3$ is in accord with the prediction of the renormalization-group $\epsilon$ expansion to the marginal orders in $\epsilon{ }^{16,36}$ The issue of the percolation constant $\mathcal{C}^{28,31}$ is intimately connected to the widely-known Alexander-Orbach conjecture,${ }^{24}$ which up to recently has been amongst the biggest open challenges in the fundamental percolation theory. Note, also, that Eq. (12) is restricted to contractible ${ }^{32}$ fractal sets; the noncontractibility effects tend to diminish the actual value of the spectral fractal dimension $d_{s}$ at $q=q_{c}$ when compared to the percolation constant $\mathcal{C} \approx 1.327 .{ }^{28,31}$

In view of Eq. (12), the fracton DOS near criticality becomes

$$
\mathcal{D}_{\mathrm{fr}}(\omega) \propto \omega^{\mathcal{C}-1} \sim \omega^{1 / 3},
$$

where the mean-field estimate $\mathcal{C} \sim 4 / 3$ has been used for simplicity. From Eqs. (8), (9) and (13) one concludes that the fracton states $\left(d_{s}=\mathcal{C} \sim 4 / 3\right)$ are much denser than the phonon states $\left(d_{s}=d \geq 2\right)$ for the frequencies $\omega$ smaller than $\sim \omega_{\min } \times(\xi / a)^{\sigma}$. Fractons could thereby dominate in the excitation spectrum of the system below the characteristic temperature of

$$
T_{c} \sim \hbar \omega_{\min } \times(\xi / a)^{\sigma} \sim 2 \pi \hbar(c / a) \times(\xi / a)^{\theta / 2},
$$

in accordance with condition (7).

\section{TOPOLOGICAL CONSTRAINTS ON THE HAUSDORFF DIMENSION}

In this section, topological constraints on the Hausdorff dimension $d_{f}$ are found. The results obtained are further used to define the admissible values of the index of connectivity $\theta$ and of the exponent $\sigma=(2+\theta) / 2$ in Eq. (14).

First, let us observe that the closed fat percolating network $\overline{\mathcal{N}}_{c}=\phi^{-1} \bar{D}^{d_{s}}$ can be densely covered everywhere by a fractal curve $\Gamma^{*}=\phi^{-1} \gamma^{*}$ which has no points of self-crossings. (Remind the diffeomorphism $\left\{\phi: \partial \mathcal{N}_{c} \rightarrow S^{d_{s}-1}, \mathcal{N}_{c} \rightarrow D^{d_{s}}\right\}$ between the closed fat percolating network $\overline{\mathcal{N}}_{c}=\mathcal{N}_{c} \bigcup \partial \mathcal{N}_{c}$ and the fractional manifold $\bar{D}^{d_{s}}=D^{d_{s}} \cup S^{d_{s}-1}$.) We now prove that the Hausdorff fractal dimension, $\operatorname{dim} \Gamma^{*}$, of the curve $\Gamma^{*}$ is equal to $d_{f} \equiv \operatorname{dim} \partial \mathcal{N}_{c},{ }^{18}$ i.e.,

$$
d_{f} \equiv \operatorname{dim} \partial \mathcal{N}_{c}=\operatorname{dim} \Gamma^{*} .
$$

In fact, because $\Gamma^{*}$ is dense everywhere in $\overline{\mathcal{N}}_{c}$, one has $\operatorname{dim} \Gamma^{*}=\operatorname{dim} \overline{\mathcal{N}}_{c}$. We need to demonstrate that the critical percolating network $\partial \mathcal{N}_{c}$ is dense everywhere in the closed fat percolating network $\overline{\mathcal{N}}_{c}$ : This would imply $\operatorname{dim} \partial \mathcal{N}_{c}=\operatorname{dim} \overline{\mathcal{N}}_{c}$, thus leading to Eq. (15).

Lemma: $\partial \mathcal{N}_{c}$ is dense everywhere in $\overline{\mathcal{N}}_{c}$. This means, precisely, that for arbitrary $\varepsilon>0$ and arbitrary point $A_{c} \in \mathcal{N}_{c}$ one finds another point $A_{c}^{*} \in \partial \mathcal{N}_{c}$, such that the distance $\left\|A_{c}-A_{c}^{*}\right\| \leq \varepsilon{ }^{33}$ [Roughly speaking, the 
(open) fat percolating network $\mathcal{N}_{c}$ has an "extremely developed" boundary $\partial \mathcal{N}_{c}$ at $q=q_{c}$.] Note that $\|\ldots\|$ is Euclidean distance in the embedding $d$-dimensional space. Assume the contrary: $\partial \mathcal{N}_{c}$ is not dense everywhere in $\overline{\mathcal{N}}_{c}$. Hence there exist $\varepsilon>0$ and $A_{c} \in \mathcal{N}_{c}$, such that $A_{c}$ can be surrounded by the closed $(d \geq 2)$ dimensional Euclidean $\varepsilon$-disk $\bar{D}_{\varepsilon}^{d}$ which lies in $\mathcal{N}_{c}$ but contains no points of the boundary $\partial \mathcal{N}_{c}$, i.e., $\bar{D}_{\varepsilon}^{d} \subset \mathcal{N}_{c}$, $\bar{D}_{\varepsilon}^{d} \bigcap \partial \mathcal{N}_{c}=\emptyset$. Because $\Gamma^{*}$ densely covers $\overline{\mathcal{N}}_{c}^{\varepsilon}$ everywhere, the $\varepsilon$-element $\Gamma_{\varepsilon}^{*}=\Gamma^{*} \bigcap \bar{D}_{\varepsilon}^{d}$ densely covers $\bar{D}_{\varepsilon}^{d}$ everywhere for $d \geq 2$. Remark that $\Gamma_{\varepsilon}^{*}$ has no points of self-crossings. Consequently, we constructed a dense covering everywhere of a $(d \geq 2)$-dimensional Euclidean domain $\bar{D}_{\varepsilon}^{d}$ by a fractal curve $\Gamma_{\varepsilon}^{*}$ which has no points of self-crossings. This, however, is principally impossible in $d \geq 2$ dimensions. ${ }^{37}$ For instance, a dense covering everywhere of a two-dimensional $(d=2)$ Euclidean domain $\bar{D}_{\varepsilon}^{2}$ is provided by the Peano curve which has infinite number of points of self-crossings (e.g., of multiplicity 4) where different segments of the curve are in contact with each other. ${ }^{37}$ The observed contradiction proves the lemma.

The next step is to consider general embedding conditions for the curves that have no points of self-crossings. Indeed, a plane $(d=2)$ curve can be embedded without self-crossings into at most $\mathcal{S}_{2}=\ln 8 / \ln 3 \approx 1.89<2$ dimensions, ${ }^{37}$ where $\mathcal{S}_{2} \equiv \ln 8 / \ln 3$ is the Hausdorff fractal dimension of the square Sierpinski carpet. ${ }^{14,38}$ (The inequality $\mathcal{S}_{2}<2$ excludes existence of the Peano curves without self-crossings.) Analogously, a spatial $(d=3)$ curve can be embedded without self-crossings into no more than $\mathcal{S}_{3}=\ln 26 / \ln 3 \approx 2.96<3$ dimensions, ${ }^{37,38}$ where $\mathcal{S}_{3} \equiv \ln 26 / \ln 3$ is the Hausdorff fractal dimension of the three-dimensional Cantor cheese. The existence of an embedding into the Sierpinski carpet (Cantor cheese) for the given plane (spatial) curve is a topologically invariant property ${ }^{37}$ that does not depend on the way the curve is folded in space. An application of this property to the fractal curve $\Gamma^{*}$ leads to the following conditions: $1 \leq \operatorname{dim} \Gamma^{*} \leq \mathcal{S}_{2}$ for $d=2 ; 1 \leq \operatorname{dim} \Gamma^{*} \leq \mathcal{S}_{3}$ for $d=3$. In arbitrary dimensions $d \geq 2$, one gets, accordingly

$$
1 \leq \operatorname{dim} \Gamma^{*} \leq \mathcal{S}_{d} \equiv \ln \left(3^{d}-1\right) / \ln 3<d,
$$

where $\mathcal{S}_{d} \equiv \ln \left(3^{d}-1\right) / \ln 3$ is the Hausdorff dimension of the Cantor cheese in $E^{d} \cdot{ }^{38}$ Considering Eq. (15), one finds

$$
1 \leq d_{f} \leq \mathcal{S}_{d} \equiv \ln \left(3^{d}-1\right) / \ln 3
$$

where $2 \leq d \leq 5$ due to the limitations imposed by the Whitney embedding theorem. ${ }^{31}$ Inequality (17) establishes the admissible values of the Hausdorff dimension $d_{f}$ for percolating networks at criticality $(2 \leq d \leq 5)$. From Eqs. (12) and (17) we finally obtain

$$
\begin{gathered}
-2(\mathcal{C}-1) / \mathcal{C} \leq \theta \leq 2\left(\mathcal{S}_{d}-\mathcal{C}\right) / \mathcal{C} \\
1 / \mathcal{C} \leq \sigma \leq \mathcal{S}_{d} / \mathcal{C}
\end{gathered}
$$

Inequalities (18)-(19) hold for embedding dimensions $2 \leq d \leq 5$. From Eqs. (2) and (12) one also gets

$$
\partial \theta / \partial T=2 \partial \sigma / \partial T \geq 0
$$

provided the system is at the threshold of percolation.

\section{FRACTIONAL WAVE EQUATION FOR FRACTON EXCITATIONS}

We now turn to a calculation of the fracton wave function, $\Psi(t, x)$, in a quantum state with angular frequency $\omega$ and wave vector $q$. In the harmonic approximation, we have $\Psi(t, x)=\psi(x) e^{i \omega t}$. The real-space coordinate $x$ is chosen in the direction of $q$. The standard normalization condition

$$
\int_{-\infty}^{+\infty}|\Psi(t, x)|^{2} d x=\int_{-\infty}^{+\infty}|\psi(x)|^{2} d x=1
$$

is implied. The Planck's constant $\hbar=1$ in this section.

We start from the fracton dispersion relation $\omega=\Lambda q^{\sigma}$ with $\Lambda \sim c \times(\xi / 2 \pi)^{\theta / 2}$, in accordance with Eqs. (3) and (5). Replacing $\omega$ and $q$ by the operators $i \partial / \partial t$ and $-i \nabla \equiv-i \partial / \partial x$, respectively, and taking into account the harmonic form $\Psi(t, x)=\psi(x) e^{i \omega t}$, one arrives at the fractional kinetic equation

$$
i \partial \Psi(t, x) / \partial t=i^{-\sigma} \Lambda \nabla^{\sigma} \Psi(t, x),
$$

where $\nabla^{\sigma}$ denotes the $\sigma$-th power of $\nabla$ (to be considered as the fractional generalization of the Laplacian, $\left.\Delta \equiv \nabla^{2}\right)$. An explicit analytical representation of the fractional derivative $\nabla^{\sigma}$ is given by the Riesz operator defined in Ref. ${ }^{39}$ The introduction of the fractional Eq. (22) for the (quasi)acoustic excitations in fractal networks follows the paradigm of the strange (or fractional) dynamics ${ }^{40}$ which has come of age as a complementary tool in the description of anomalous kinetic processes in complex systems. ${ }^{39,41-45}$

The real-space counterpart, $\psi(x)$, of the fracton wave function $\Psi(t, x)=\psi(x) e^{i \omega t}$ obeys the fractional equation

$$
\omega \psi(x)=-i^{-\sigma} \Lambda \nabla^{\sigma} \psi(x) .
$$

General analytical solution to Eq. (23) can be expressed in terms of Mittag-Leffler functions (these being a special case of Fox functions ${ }^{39}$ ). Elementary-function solutions may be obtained in the following limiting cases:

1) Core region: $\omega x^{\sigma} \lesssim \Lambda$ :

$$
\begin{gathered}
\psi_{n}(x) \sim \exp \left(-i^{\sigma} \frac{\omega x^{\sigma}}{\Lambda \Gamma(1+\sigma)}\right), \\
\Psi_{n}(t, x) \sim e^{i \omega t} \exp \left(-i^{\sigma} \frac{\omega x^{\sigma}}{\Lambda \Gamma(1+\sigma)}\right),
\end{gathered}
$$




$$
\left|\Psi_{n}(t, x)\right|^{2}=\left|\psi_{n}(x)\right|^{2} \sim \exp \left(-\frac{2 \omega \alpha_{n} x^{\sigma}}{\Lambda \Gamma(1+\sigma)}\right),
$$

where $i^{\sigma} \equiv \cos [\pi \sigma(4 n+1) / 2]+i \sin [\pi \sigma(4 n+1) / 2], n=$ $0, \pm 1, \pm 2, \ldots$ is an integer number, and $\alpha_{n}=\cos [\pi \sigma(4 n+$ $1) / 2$ ] is the real part of $i^{\sigma}$. Admissible (decaying) fracton modes $\Psi_{n}(t, x)$ are selected by the condition

$$
\alpha_{n}=\cos [\pi \sigma(4 n+1) / 2] \geq 0 .
$$

The shape of the fracton wave function in the core region $\omega x^{\sigma} \lesssim \Lambda$ is thereby a stretched exponential distribution $\psi_{n}(x) \sim \exp \left[-\left(x / \lambda_{n}\right)^{\sigma}\right]$, with $\lambda_{n}^{\sigma}=\Lambda \Gamma(1+\sigma) / \omega \alpha_{n}$. The spatial scale $\lambda_{n}$ (i.e., the size of the core region) is often referred to as the fracton localization length. ${ }^{25}$ Note that the values of $\sigma$ lie in the interval (19) and may depend on the embedding dimension $2 \leq d \leq 5$ in general. Numerically, $0.75 \lesssim \sigma \lesssim 1.42$ for $d=2$, and $0.75 \lesssim \sigma \lesssim 2.23$ for $d=3$. In Euclidean geometries, $\sigma=1$, yielding $\alpha_{n} \equiv 0$ for all $n$. In this limit, the fracton wave function $\Psi_{n}(t, x)$ crosses over to a plane wave, $\sim \exp (i \omega t-i q x)$.

The stretched exponential behavior of the fracton wave function in the core region was first proposed by EntinWohlman et al. ${ }^{46}$ Much theoretical and numerical effort has later been invested to determine the value of $\sigma$ (for a review, see Nakayama et al. ${ }^{16}$ and references therein. In the notations of article, ${ }^{16} \sigma \equiv d_{\phi}$.) The results addressed in Ref. ${ }^{16}$ comply well with the constraint (19) in both cases $d=2$ and $d=3$.

2) Tail region: $\omega x^{\sigma} \gg \Lambda$ : The asymptotic $\left(\omega x^{\sigma} \gg \Lambda\right)$ analysis of the fractional Eq. (23) is similar to a derivation of the Lévy stable distributions ${ }^{39}$ from the Riesz operator. Making use of the normalization condition (21), from Eq. (23) one finds [cf. Eqs. (24)-(26)]

$$
\begin{gathered}
\psi_{n}(x) \sim\left(\frac{\Lambda}{\omega \Gamma(-\sigma)}\right)^{1 / 2} \times x^{-(\sigma+1) / 2}, \\
\Psi_{n}(t, x) \sim\left(\frac{\Lambda}{\omega \Gamma(-\sigma)}\right)^{1 / 2} e^{i \omega t} \times x^{-(\sigma+1) / 2}, \\
\left|\Psi_{n}(t, x)\right|^{2}=\left|\psi_{n}(x)\right|^{2} \sim \frac{\Lambda}{\omega|\Gamma(-\sigma)|} \times x^{-(\sigma+1)} .
\end{gathered}
$$

Equation (30) represents the power-law tail of the fracton wave function $\Psi_{n}(t, x)$, with the exponent $\sigma+1$ varying from approximately 1.75 to $\approx 2.42$ in two dimensions $(d=2)$, and up to $\approx 3.23$ in three dimensions $(d=$ $3)$. These relatively small values of the power exponent $\sigma+1 \lesssim 3.5$ allow for the considerable probabilities of finding a fracton beyond the core region when compared to a stretched exponential interpolation. We emphasize that the power-law tail (30) is the fractional dynamics property contained in the Riesz operator $\nabla^{\sigma}$.

\section{THE INTERACTION HAMILTONIAN}

Similar to the ordinary phonons in regular crystals, fractons may mediate electron-electron interactions in fractal media. The implication is that an electron placed on a fractal network generates a structural deformation which affects another electron. The process is described by the interaction Hamiltonian

$$
\mathcal{H}=\sum_{\mathbf{k}, \mathbf{p}} \mathcal{W}_{\mathbf{k p}} \phi_{\mathbf{p}-\mathbf{k}} a_{\mathbf{p}}^{+} a_{\mathbf{k}}+\sum_{\mathbf{k}, \mathbf{p}} \mathcal{W}_{\mathbf{k} \mathbf{p}}^{*} \phi_{\mathbf{p}-\mathbf{k}}^{+} a_{\mathbf{k}}^{+} a_{\mathbf{p}}
$$

where $a_{\mathbf{k}}^{+}$is the creation operator for an electron with wave vector $\mathbf{k}, \phi_{\mathbf{p}-\mathbf{k}}^{+}$is the creation operator for a fracton with wave vector $\mathbf{p}-\mathbf{k}$, and $\mathcal{W}_{\mathbf{k p}}$ are the elements of the interaction matrix, maximized for $\mathbf{p}=-\mathbf{k}$. The fracton operators $\phi_{\mathbf{p}-\mathbf{k}}^{+}$and $\phi_{\mathbf{p}-\mathbf{k}}$ are introduced for the excitations that obey the selection rule (27). The Hamiltonian in Eq. (31) recovers the conventional electronphonon interaction Hamiltonian ${ }^{30}$ in the Euclidean geometry limit $d_{f} \rightarrow d$ and $d_{s} / d_{f} \rightarrow 1$. The existence of the electron coupled states for the Hamiltonian (31) can be demonstrated following the standard formalism. ${ }^{9,30}$ Such states would physically correspond to the "Cooper pairs" on fractal networks. Like in the conventional, phononmediated picture of electron-electron interactions, ${ }^{9,30}$ the Cooper pairs in fractal geometry occur because electrons with nearly opposite momenta $(\mathbf{k},-\mathbf{k})$ exchange a fracton excitation.

Owing to the effect of the fractional dynamics, the electron-fracton interactions are not restricted to the core region $\omega x^{\sigma} \lesssim \Lambda$, but are extended, by means of the Riesz operator $\nabla^{\sigma}$, to the "strange" domain $\omega x^{\sigma} \gg \Lambda$ corresponding to the power-law tail of the fracton wave function. The tail counterpart given by Eqs. (28)-(30) supports broad electron-electron couplings in the medium, enabling the formation of the Cooper pairs throughout the self-similarity interval $a \lesssim \chi \lesssim \xi$. In view of inequality (20), the couplings are more extensive at lower absolute temperatures $T$. In view of the high density of states in Eq. (13) (large compared to the phonon DOS), fracton excitations beneficiate as a favourable ingredient of the superconducting transition in strange materials.

\section{TUNNELING EFFECTS}

In fractal networks, the onset of superconductivity may be limited to the Anderson localization of the Cooper pairs. In fact, as it follows from inequalities (2) and (20), the fractal geometry of the network creates a potential barrier for a particle, with the height proportional to $T$. A superconducting behavior on a network structure could be the case if the quantum tunneling length for the charge carriers exceeds the characteristic Anderson localization length on the fractal. The Anderson localization length, in its turn, is typically of the order of the microscopic 
distance $a$, describing the characteristic scale of the inhomogeneities present. The quantum tunneling length for a particle of mass $m^{*}$ is given by the well known estimate $l^{*} \sim \hbar / \sqrt{2 m^{*} T}$, where $T$ stands for the height of the barrier. A transition to a superconducting state may thereby occur if $l^{*} \gtrsim a$. Taking into account that $m^{*}=2 m_{e}$ for the Cooper pairs, one finds the appropriate temperatures to be

$$
T \lesssim \hbar^{2} / 4 m_{e} a^{2}
$$

(We imply that the material is well supplied with the free electrons that may effectively participate in the pairing processes. In the experiments in Ref., ${ }^{5}$ the necessary charge carrier concentration was achieved through the gate-induced doping in the field-effect device.) Setting $a \sim 10^{-6} \mathrm{~cm},{ }^{5}$ we have $T \lesssim(2-3) \mathrm{K}$. This complies with the onset temperature $T_{c} \sim 2.35 \mathrm{~K}$ reported in Ref. ${ }^{5}$

The tunneling of the Cooper pairs through braided molecular structures is the marginal dynamical process that limits the superconducting behavior in the polymeric systems. The corresponding temperatures $T \lesssim$ $(2-3) \mathrm{K}$ are rather modest owing to the relatively large threshold tunneling length $l^{*} \sim a \sim 10^{-6} \mathrm{~cm}$ on the right of inequality (32). [Decreasing this length might promise enhanced temperatures, of course.] Anticipating the results of the next Section, we note that the fractonmediated superconductivity may come into play at the considerably higher temperatures (typically, as high as $\left.T_{c} \sim 150 \mathrm{~K}\right)$, provided the tunneling limitation (32) is circumvented.

The tunneling processes on a fractal set could be associated with the formation of a disordered array of Josephson junctions enveloping the domains of effective electron-fracton interactions. In this context, it is worth noting that superconductivity, due to Josephson coupling of the superconducting domains, was in fact observed in polycrystalline systems. ${ }^{47}$ The role of Josephson arrays for the superconducting behavior in P3HT was addressed in Ref. ${ }^{5}$ in connection with the patchy (granular) topology of the transition regions.

The fracton pairing mechanism might explain why superconductivity has not been observed in inorganic semiconductor devices, ${ }^{7}$ such as $\mathrm{Si}$ and GaAs: The active materials used in such devices lack the structural complexity which might support a well-defined branch of fracton excitations in the self-similar domain. Conversely, selfassembling organic polymers like P3HT reveal a complex organization owing to SOC, that offers a fertile playground for the fracton modes as soon as the criticality condition (12) is satisfied.

\section{A CASE FOR HIGH-TEMPERATURE SUPERCONDUCTIVITY}

An important ramification of the present study is a model when electrons are replaced by charged "holes" participating in the fracton exchange processes on a complex substrate. The simplest interaction Hamiltonian reads

$$
\mathcal{H}=\sum_{\mathbf{k}, \mathbf{p}} \mathcal{W}_{\mathbf{k p}} \phi_{\mathbf{p}-\mathbf{k}} b_{\mathbf{p}}^{+} b_{\mathbf{k}}+\sum_{\mathbf{k}, \mathbf{p}} \mathcal{W}_{\mathbf{k} \mathbf{p}}^{*} \phi_{\mathbf{p}-\mathbf{k}}^{+} b_{\mathbf{k}}^{+} b_{\mathbf{p}}
$$

Here, $b_{\mathbf{k}}^{+}$and $b_{\mathbf{k}}$ denote the creation-annihilation operators for a hole with wave vector $\mathbf{k}$. The expression (33) is analogous to the Hamiltonian in Eq. (31) for the fractonmediated electron coupling. The interaction processes (33) could dominate in the nominally insulating materials at high hole-doping levels. Beside hole-doped systems, the hole coupling effects might be essential in cuprate superconductors owing to the specific crystalline structure of the copper-oxide compounds. In fact, the cuprate superconductors consist of parallel planes of copper and oxygen atoms arranged in a square grid. The copperoxygen planes are separated by the layered atoms of other elements, which may absorb electrons from the copper sites, leaving positively charged holes behind. ${ }^{48}$ The integral system operates like a multi-layer field-effect transistor with the conducting copper-oxygen planes confined between the charge-absorbing insulating substrates. The complex microscopic texture of the compound supports fractional harmonic modes in Eq. (22) that may turn the holes into coupled states.

The key point about the holes is that they have negative mass $m^{*}=-\left|m^{*}\right|<0$, leading to the imaginary fractal tunneling length $l^{*} \sim i \hbar / \sqrt{2\left|m^{*}\right| T}$. This means periodic charge oscillations $\sim \exp \left(-i x \sqrt{2\left|m^{*}\right| T} / \hbar\right)$ in the copper-oxygen lattice, i.e., a "stripe". ${ }^{48}$ The stripes form a runway ${ }^{48}$ along which the coupled holes may flow without resistance. The stripe order can be associated with self-organization of the system to a thermodynamically profitable one-dimensional charge distribution.

It is fair to remark that the issue of stripes is at present widely debated in the literature. Theoretical arguments in favour of the stripes have been proposed in Ref. ${ }^{49}$ Concerns come from the poor observational support for the charge stripes as an integral part of the superconducting behavior in the cuprates. Intriguing experimental indications of the charge-stripe order in high-temperature superconductors have been obtained only recently. ${ }^{50,51}$ (See, also, Ref. ${ }^{52}$ where an evidence for the stripes in a chemically doped cuprate compound was reported.)

Inclusion of stripes makes it possible to bypass the marginal limitation (32) on the superconducting temperature range. The typical superconducting transition temperature $T_{c}$ can then be directly evaluated from expression (14), provided the charge is carried by the fracton coupled holes along the stripe runways. Considering condition (20), we maximize the index of connectivity $\theta$ in the scaling $T_{c} \propto(\xi / a)^{\theta / 2}$ to be $\theta=\theta_{\max }=$ $2\left(\mathcal{S}_{d}-\mathcal{C}\right) / \mathcal{C}$, where $\mathcal{S}_{d}$ is the Hausdorff dimension of the Cantor cheese $\mathrm{e}^{14,38}$ in $E^{d}$, and $\mathcal{C}$ is the percolation constant. $^{28,31}$ (High-temperature regime is implied, enabling one to set $\theta=\theta_{\max }$ from $\partial \theta / \partial T \geq 0$.) In view 
of the plane $(d=2)$ geometry of the conducting layers in cuprate superconductors, ${ }^{48}$ we find for the scaling exponent, $\theta / 2=\theta_{\max } / 2=\left(\mathcal{S}_{2}-\mathcal{C}\right) / \mathcal{C} \approx 0.43$, where $\mathcal{S}_{2} \approx 1.89$ is the Hausdorff dimension of the square Sierpinski carpet. ${ }^{14,38}$ Assuming further the maximal ${ }^{20}$ ratio $\xi / a \sim 10^{3}$, we estimate the factor $(\xi / a)^{\theta / 2}$ in Eq. (14) to be around 20. After all, making use of the characteristic values $c \sim 2 \times 10^{5} \mathrm{~cm} / \mathrm{s}$ for the long-wave phonon phase velocity, and $a \sim 10^{-6} \mathrm{~cm}$ for the minimal fracton wavelength, we have, by order of magnitude, $T_{c} \sim 40 \pi \hbar c / a \sim 150 \mathrm{~K}$. This result is in good agreement with the typical transition temperatures observed in cuprate superconductors. Enhanced transition temperatures might be speculated for the compounds having $2 \pi c / a \gtrsim 10^{12} \mathrm{~Hz}$.

We emphasize that the high transition temperature $T_{c} \sim 150 \mathrm{~K}$ profits from the imaginary hole tunneling length, $l^{*} \sim i \hbar / \sqrt{2\left|m^{*}\right| T}$, giving rise to the selforganized charge-stripe order across the conducting set. In cuprate superconductors, this macroscopic order helps coupled holes run freely along the copper-oxygen planes. Conversely, electrons placed on a complex conducting lattice encount multiscale potential barriers, whose height grows with absolute temperature $T$. (The effect of the barriers could be strengthened in the cuprates because of the electron absorbtion processes.) "Loaded" with the positive mass (opposite to that of holes), electrons can enter into a superconducting state at the low enough temperatures $T \lesssim \hbar^{2} / 4 m_{e} a^{2}$, in accordance with condition (32). Support for this viewpoint can be found in the fieldeffect doping experiments with $\mathrm{CaCuO}_{2}$ compound: ${ }^{53}$ While electron-type doping has shown the maximum value of $T_{c}$ around $34 \mathrm{~K}$, hole-type doping revealed an enhanced superconducting transition temperature $T_{c}$ near $89 \mathrm{~K}$. Note that the field-effect geometry exploited in the experiments in Ref. ${ }^{53}$ mimics the inherent microscopic organization of the copper oxides composed of the alternating conducting-insulating layers.

We suggest that fracton coupled holes is the most probable ingredient of the high-temperature superconducting phase in copper oxides. This proposal is somewhat different from the theoretical results of Buttner and Blumen, ${ }^{54}$ who argued that such a phase might directly occur from the fracton-mediated electron-electron interactions. Nevertheless, the focus on fractons ${ }^{54}$ as the effective glue that may confine the current-carrying particles in couples on a complex geometry is in accord with the basic ideas of our study. Intrinsic multiscale inhomogeneities that might support the fracton modes in high-temperature superconductors have been studied experimentally in Ref. ${ }^{55}$

The direct observation of the fracton-hole interactions in the cuprate superconductors is an open challenge. Such interactions might be recognized from meandering of the charge stripes, a phenomenon addressed in Ref. ${ }^{49}$ The meandering property has been strongly advocated in Ref. ${ }^{51}$ from the novel in-plane transport anisotropy in copper oxides.

\section{AC UNIVERSALITY}

The complex percolating lattices underlying the fracton excitation spectrum (13) may leave an imprint on the ac conduction properties of the material above the superconducting transition temperature $T_{c}$. As is shown in Ref., ${ }^{18}$ self-organization of the medium to a state dominated by the critical percolation structures results in the universal behavior of ac conduction at the "anomalous" frequencies $\Omega$, for which the charge carriers walk on the fractal. The defining feature of ac universality is independence of the microscopic details of the pattern on which the random walk takes place, and of the nature of the conducting mechanism operating in the system (i.e., classical barrier crossing for ions and/or quantummechanical tunneling for electrons). Ac universality can be represented analytically by the power-law dependence of the conductivity $\Sigma(\Omega)$ versus frequency $\Omega:^{23}$

$$
\Sigma(\Omega) \propto \Omega^{\eta} .
$$

For the bulk conduction, the exponent $\eta$ lies typically between 0.6 and 1.0. ${ }^{23}$ Among other materials, the bulk ac universality was recognized in ion and electron conducting polymers, amorphous and polycrystalline semiconductors, organic-inorganic composites, doped semiconductors at helium temperatures, etc. (see Refs. ${ }^{23}$ and $^{56}$, and references therein).

Ac universality (34) results from the self-similar behavior of the random walk processes on the fractal geometry. ${ }^{18}$ Given a path-connected fractal distribution with connectivity $\theta$, one finds the mean-square displacement of the walker (e.g., of the charged particle placed on the disordered conducting array) after time $t$ to be ${ }^{12}$

$$
\left\langle\delta \chi^{2}(t)\right\rangle \propto t^{2 /(2+\theta)} \quad(a \lesssim|\delta \chi| \lesssim \xi) .
$$

The probability to return to the starting point ${ }^{27}$ scales with time as $p(t) \propto t^{-d_{s} / 2}$, where $d_{s}$ is the spectral dimension of the fractal, which simultaneously arises in the fracton DOS in Eq. (8). Near the threshold of percolation, $d_{s}=\mathcal{C}$ and $p(t) \propto t^{-\mathcal{C} / 2}$. In the limit of Euclidean geometry $\left(d_{s} \rightarrow d, \theta \rightarrow 0\right)$, Eq. (35) recovers the conventional Einstein law $\left\langle\delta \chi^{2}(t)\right\rangle \propto t$ for the particle diffusion. Generalized transport equations that account for the "strange" 40 relation (35) have been considered in Refs. $^{22,27,39,41,57}$

Based on the self-similar random walk model (35), Gefen et al. ${ }^{12}$ demonstrated that ac conductivity of percolating fractal networks behaves as a power of frequency, $\Sigma(\Omega) \propto \Omega^{\eta}$, with the exponent $\eta=\mu / \nu(2+\theta)$. This is in accord with the dependence (34) customarily observed in real disordered materials. Note that the quantity $\eta$ is a combination of the indices $\mu, \nu$, and $\theta$, which mirror the percolative nature of the underlying fractal lattice. Making use of the expressions ${ }^{12} d_{f}=d-\beta / \nu$ and $\theta=(\mu-\beta) / \nu$, one finds

$$
\eta=\left(\theta+d-d_{f}\right) /(2+\theta) .
$$


Equation (36) implies that the dependence $\Sigma(\Omega)$ is controlled by the characteristic distance $|\delta \chi|$ traveled by a particle during the time $1 / \Omega$. Considering expression (35), one arrives at the scaling $\Omega \propto|\delta \chi|^{-(2+\theta)}$ which relates the applied frequency $\Omega$ to the displacement $|\delta \chi|$.

For sufficiently low frequencies $\Omega \lesssim \xi^{-(2+\theta)}$, the current-carrying particles cover distances $|\delta \chi| \gtrsim \xi$ during the period of $1 / \Omega$. In this limit, the charge carriers feel statistically homogeneous distribution of the conducting links, corresponding to $d_{f} \rightarrow d$ and $\theta \rightarrow 0$. From Eq. (36) one concludes that $\eta \rightarrow 0$ for $\Omega \lesssim \xi^{-(2+\theta)}$. Hence $\Sigma(\Omega) \propto \Omega^{\eta}$ is almost frequency independent in the lower frequency range: $\Sigma(\Omega) \rightarrow \Sigma(0)=$ const $(\Omega)$ for $\Omega \lesssim \xi^{-(2+\theta)}$, where $\Sigma(0)$ is dc conductivity.

For $\xi^{-(2+\theta)} \lesssim \Omega \lesssim a^{-(2+\theta)}$, the charge transport processes are governed by the self-similar (fractal) geometry of the underlying conducting set. In this regime, the value of $\eta$ obeys the topological constraints imposed by the percolation threshold character. In fact, combining Eqs. (12) and (36), we get $(2 \leq d \leq 5)$ :

$$
\eta=(2-\mathcal{C}) / 2+(d-2) \mathcal{C} / 2 d_{f}
$$

For the plane lattices $(d=2)$, the term depending on the Hausdorff dimension $d_{f}$ vanishes. Expression (37) is then reduced to the "hyperuniversal" ${ }^{18}$ counterpart

$$
\eta=(2-\mathcal{C}) / 2 \approx 0.34 \sim 1 / 3 \quad(d=2),
$$

involving solely the percolation constant $\mathcal{C}$. The exponent in Eq. (38) describes ac conduction of thin films of complex materials revealing a two-dimensional $(d=2)$ self-similar organization. Ac conductivity of the selfsimilar (fractal) films scales with frequency $\xi^{-(2+\theta)} \lesssim$ $\Omega \lesssim a^{-(2+\theta)}$ as $\Sigma(\Omega) \propto \Omega^{1 / 3}$, where we neglected the small deviation of the percolation constant $\mathcal{C} \approx 1.327$ from the mean-field value $4 / 3$.

The "hyperuniversal" ${ }^{18}$ behavior $\Sigma(\Omega) \propto \Omega^{1 / 3}$ in two dimensions should be considered as a specific prediction of our model: To our best knowledge, reliable experimental data on ac conduction of the fractal films are almost absent in the literature. Direct observational verification of the scaling $\Sigma(\Omega) \propto \Omega^{1 / 3}$ might be performed, e.g., through a charge injection in the field-effecttransistor geometry using the pioneering methods discussed in Refs. ${ }^{4,5,53}$

In higher dimensions $(3 \leq d \leq 5)$, the "Hausdorff" term $(d-2) \mathcal{C} / 2 d_{f}$ on the right of expression (37) apparently contributes to the exponent $\eta$. Considering the constraint (17) on the parameter $d_{f}$, we locate the value of $\eta$ in the interval $\eta_{\min } \leq \eta \leq \eta_{\max }$, with

$$
\begin{gathered}
\eta_{\min }=(2-\mathcal{C}) / 2+(d-2) \mathcal{C} / 2 \mathcal{S}_{d}, \\
\eta_{\max }=1+(d-3) \mathcal{C} / 2 .
\end{gathered}
$$

Here, $\mathcal{S}_{d}=\ln \left(3^{d}-1\right) / \ln 3$ is the Hausdorff dimension of the Cantor cheese in $E^{d}$. In three dimensions $(d=3)$, one finds $\eta_{\min } \leq \eta \leq 1$, where $\eta_{\min }=(2-\mathcal{C}) / 2+\mathcal{C} / 2 \mathcal{S}_{3}$. Numerically, $\eta_{\min } \approx 0.56$, in close agreement with the observational result $0.6 \lesssim \eta \leq 1.0 .^{23}$

In view of inequality (2), from Eq. (37) we also get

$$
\begin{gathered}
\partial \eta / \partial T=0 \quad(d=2), \\
\partial \eta / \partial T \leq 0 \quad(d=3) .
\end{gathered}
$$

Hence, in three dimensions, $\eta$ generally increases with decreasing temperature $T$, a phenomenon often observed in real disordered materials. ${ }^{23}$

We emphasize that ac universality (34), although effective above the typical superconducting transition temperature $T_{c}$, is an integral part of the fracton scenario as it derives from the same general principles basing on the percolative geometry of the underlying conducting lattice. Ac universality might serve as an indication that the material posseses the complex enough microscopic organization that supports a wide fracton spectrum. Acuniversal materials could thereby be plausible candidates into the strange superconductors dominated by the fracton-mediated interactions between the currentcarrying particles. A test on ac universality might help verify the fracton nature of the pairing mechanism potentially operating in organic polymers and copper oxides.

\section{SUMMARY AND CONCLUSIONS}

In spite of the diverse superconducting transition temperatures, self-assembling organic polymers and copperoxide compounds bear important features in common. In fact, the polymers superconduct in the field-effecttransistor geometry at high gate-induced charge-carrier concentrations. The superconducting state is supported by self-organization mechanisms governing the kinetics of the polymer system. Owing to self-organization, the polymers form thin regular films, on which the superconducting electron fluid condensates.

The cuprate superconductors, in their turn, operate like multi-layer transistors due to the intrinsic fieldeffect crystalline configuration (i.e., conducting copperoxygen planes confined between charge-absorbing insulating substrates). Self-organization processes appear in the charge-stripe order enabling the current-carrying particles flow along the copper-oxygen planes without resistance.

Both polymers and copper oxides are "strange" superconductors as they involve unconventional (other than BCS) pairing processes underlying the superconducting phase. In either of the materials, the (quasi)particles mediating the interaction between the charge carriers are suggested to be fractons, quantum oscillations of fractal lattices.

The interaction processes involving fractons allow for larger energies than that of the phonons, leading to a possibility of the high-temperature superconducting phases 
in complex materials. For the cuprate compounds, we estimate the typical superconducting transition temperature to be around $T_{c} \sim 150 \mathrm{~K}$, in good agreement with the experimental values. We imply that the marginal ingredient of the corresponding high-temperature superconducting phase are fracton coupled holes that condensate in the conducting copper-oxygen planes owing to the specific field-effect-transistor geometry of the cuprate superconductors. For the gate-induced superconductivity in the self-assembling polymers, we find much smaller transition temperatures of $\sim(2-3) \mathrm{K}$, provided the charge carriers are fracton coupled electrons, not holes. Such a modest value is due to the limitations imposed by the electron tunneling processes on the braided polymer structures. We speculate that replacing electrons with holes helps circumvent the tunneling restrictions. The fracton model then promises enhanced onset temperatures for the hole-doped systems when compared to their electron-doped counterparts. The hint is that hole-doped complex materials whose structural characteristics meet a wide fracton spectrum, might be feasible candidates in the high-temperature superconductors. Support for this suggestion partially comes from the field-effect doping experiments discussed in Ref. ${ }^{53}$ Hole doping might thereby open a perspective on the superconducting systems having large transition temperatures $T_{c} \gtrsim 150 \mathrm{~K}$.

We found that the fracton wave function contains a power-law tail stretching far beyond the central core region. The power-law constituent supports interactions between the charge carriers throughout the self-similar (fractal) domain of the conducting system. In the harmonic approximation, the fracton wave function derives from a generalized kinetic equation with the integer time and fractional real-space derivatives. The latter is given by the Riesz operator addressing the fractional generalization of the Laplacian. The introduction of the fractional kinetic equation for fracton excitations follows the paradigm of the strange dynamics ${ }^{40}$, one of the biggest open challenges in the modern nonlinear physics. The parameters of the fracton wave function describing the shape of the core and tail regions could be obtained from the unconventional, topological analysis basing on the concept of the fractional manifold. ${ }^{31}$ The fractional kinetics approach supplied with the topological guide to fractal objects yields insight into the fundamental dynamical properties underlying fracton statistics.

The formation of self-similar (fractal) lattices associated with a well-defined branch of fracton excitations affects ac conduction properties of the material in the classical energy range (i.e., above the typical superconducting transition temperature $T_{c}$ ). Based on the topological arguments, we demonstrated the universal character of ac conduction in fractal geometries, which does not depend on the details of the lattice, nor on the nature of the microscopic conducting mechanism. This universal character is contained in the scaling $\Sigma(\Omega) \propto \Omega^{\eta}$, with the exponent $\eta$ related to the fundamental topological parameters characterizing the fractal distribution, such as the percolation constant ${ }^{31}$ and the Hausdorff dimension of the Cantor cheese. ${ }^{38}$ In three dimensions (i.e., for the bulk conduction), the value of $\eta$ varies from approximately 0.56 to 1.0 , in precise agreement with the experimental range $0.6 \lesssim \eta \leq 1.0 .^{23}$ In two dimensions (i.e., for the plane fractal lattices), a "hyperuniversal" 18 value $\eta \approx 0.34 \sim 1 / 3$ is recognized, leading to the scaling $\Sigma(\Omega) \propto \Omega^{1 / 3}$ in a wide temperature range $T \gtrsim T_{c}$. This behavior should be considered as a specific theoretical prediction of our model. Observational verification of the scaling $\Sigma(\Omega) \propto \Omega^{1 / 3}$ in plane fractal geometries might shed light on the electric properties of the complex materials.

It might be extremely interesting to verify the scaling $\Sigma(\Omega) \propto \Omega^{1 / 3}$ for cuprate oxides and self-assembling organic polymers, whose microscopic organization seems to support plane fractal lattices. An observation of the scaling $\Sigma(\Omega) \propto \Omega^{1 / 3}$ might be a strong argument in favour of the fracton-mediated pairing processes operating in the strange superconductors. Extensive studies in this direction might be strongly advocated.

We are grateful to the anonymous referee for the careful reading of this paper and for the stimulating suggestions. This study was carried out when A. V. M. stayed at Risoe on a Grant from the Graduate School in Nonlinear Science. Partial support was received from Danish Natural Science Foundation (SNF), INTAS Project 971612, and RFFI Grants 00-02-17127, 00-15-96631, and 02-02-06541.

${ }^{1}$ R. B. Laughlin, Phys. Rev. Lett. 50, 873 (1983).

2 J. G. Bednorz and K. A. Muller, Z. Phys. B 64, 189 (1986).

${ }^{3}$ J. H. Schon, Ch. Kloc, and B. Batlogg, Science 288, 2338 (2000).

${ }^{4}$ J. H. Schon, Ch. Kloc, and B. Batlogg, Nature 406, 702 (2000).

5 J. H. Schon, A. Dodabalapur, Z. Bao, Ch. Kloc, O. Schenker, and B. Batlogg, Nature 410, 189 (2001).

${ }^{6}$ D. Jerome and K. Bechgaard, Nature 410, 162 (2001).

${ }^{7}$ P. Phillips, Nature 406, 687 (2000).

${ }^{8}$ R. L. Greene, G. B. Street, and L. J. Suter, Phys. Rev. Lett. 34, 577 (1975).

${ }^{9}$ J. Bardeen, L. N. Cooper, and J. R. Schrieffer, Phys. Rev. 108, 1175 (1957).

${ }^{10}$ W. A. Little, J. Polymer Sci. C29, 17 (1970).

${ }^{11}$ T. R. Kirkpatrick and D. Belitz, Phys. Rev. Lett. 66, 1533 (1991); D. Belitz and T. R. Kirkpatrick, Phys. Rev. B 46, 8393 (1992); ibid. 58, 8214 (1998).

${ }^{12}$ Y. Gefen, A. Aharony, and S. Alexander, Phys. Rev. Lett. 50, 77 (1983).

${ }^{13}$ M. D. McGehee, E. K. Miller, D. Moses, and H. J. Heeger, in Advances in Synthetic Metals (eds. P. Bernier, S. Lefrant, and G. Bidan) (Elsevier, Amsterdam, 1999). p. 98.

14 J. Feder, Fractals (Plenum, New York, 1988). 
${ }^{15}$ D. Stauffer and A. Aharony, Introduction to Percolation Theory (Taylor and Francis, London, 1992); M. B. Isichenko, Rev. Mod. Phys. 64, 961 (1992).

16 T. Nakayama, K. Yakubo, and R. L. Orbach, Rev. Mod. Phys. 66, 381 (1994).

17 B. B. Mandelbrot, The Fractal Geometry of Nature (W. H. Freeman, New York, 1983).

18 A. V. Milovanov and J. J. Rasmussen, Phys. Rev. B 64, 212203 (2001).

19 A. Le Mèhautè, Fractal Geometries: Theory and Applications (CRC Press, Boca Raton, 1991).

${ }^{20}$ E. Courtens, R. Vacher, and E. Stoll, Physica D 38, 41 (1989).

${ }^{21}$ P. Bak, C. Tang, and K. Wiesenfeld, Phys. Rev. Lett. 59, 381 (1987); Phys. Rev. A 38, 364 (1988). H. J. Jensen, SelfOrganized Criticality. (Cambridge Univ. Press, Cambridge, 1998).

22 A. V. Milovanov, Phys. Rev. E 63, 047301 (2001).

${ }^{23}$ J. C. Dyre and T. B. Schroder, Rev. Mod. Phys. 72, 873 (2000).

${ }^{24}$ S. Alexander and R. L. Orbach, J. Phys. Lett. 43, L625 (1982).

${ }^{25}$ R. Orbach, Physica D 38, 266 (1989).

${ }^{26}$ P. W. Anderson, Phys. Rev. 109, 1492 (1958).

${ }^{27}$ B. O'Shaughnessy and I. Procaccia, Phys. Rev. Lett. 54, 455 (1985); Phys. Rev. A 32, 3073 (1985).

${ }^{28}$ A. V. Milovanov and G. Zimbardo, Phys. Rev. E 62, 250 (2000).

29 J.-P. Bouchaud and A. Georges, Phys. Rep. 195, 12 (1990).

${ }^{30}$ R. P. Feynman, Statistical Mechanics (Benjamin, Massachusetts, 1972).

31 A. V. Milovanov, Phys. Rev. E 56, 2437 (1997).

${ }^{32}$ Ch. Nash and S. Sen, Topology and Geometry for Physicists (Academic, London, 1983); A. T. Fomenko and D. B. Fuks, A Course of Homotopic Topology (Nauka, Moscow, 1989).

33 J. L. Kelley, General Topology (Van Nostrand, Princeton, NJ, 1957)

${ }^{34}$ B. A. Dubrovin, S. P. Novikov, and A. T. Fomenko, Modern Geometry. Methods and Applications (Nauka, Moscow, 1986).

${ }^{35}$ M. W. Hirsch, Differential Topology (Springer-Verlag, New York, 1976).

${ }^{36}$ S. Havlin and D. ben-Avraham, Adv. Phys. 36, 695 (1987). D. ben-Avraham and S. Havlin, Diffusion and Reactions in Fractals and Disordered Systems (Cambridge Univ. Press, Cambridge, 2000).

${ }^{37}$ V. G. Boltyanskyi and V. A. Efremovich, Descriptive Topology (Nauka, Moscow, 1983).

${ }^{38}$ M. Schroeder, Fractals, Chaos, Power Laws (W. H. Freeman, New York, 1991).

${ }^{39}$ R. Metzler and J. Klafter, Phys. Rep. 339, 1 (2000).

${ }^{40}$ M. F. Shlesinger, G. M. Zaslavsky, and J. Klafter, Nature (London) 363, 31 (1993).

${ }^{41}$ G. M. Zaslavsky, Physica D 76, 110 (1994).

42 J. Klafter, M. F. Shlesinger, and G. Zumofen, Phys. Today 49, 33 (1996).

${ }^{43}$ G. M. Zaslavsky, Physica A 288, 431 (2000).

${ }^{44}$ G. M. Zaslavsky, M. Edelman, H. Weitzner, B. Carreras, G. McKee, R. Bravenec, and R. Fonck, Phys. Plasmas 7, 3691 (2000).
45 A. V. Milovanov and L. M. Zelenyi, Phys. Rev. E 64, 052101 (2001).

${ }^{46}$ O. Entin-Wohlman, S. Alexander, and R. L. Orbach, Phys. Rev. B 32, 8007 (1985).

${ }^{47}$ E. Simanek, Inhomogeneous Superconductors: Granular and Quantum Effects (Oxford Univ. Press, New York, 1994).

48 A. Cho, Phys. Rev. Focus, 27 February 2002.

${ }^{49}$ S. A. Kivelson, E. Fradkin, and V. J. Emery, Nature (London) 393, 550 (1998); E. W. Carlson, D. Orgad, S. A. Kivelson, and V. J. Emery, Phys. Rev. B 62, 3422 (2000).

${ }^{50}$ H. A. Mook, P. Dai, and F. Dogan, Phys. Rev. Lett. 88, 097004 (2002).

${ }^{51}$ Y. Ando, K. Segawa, S. Komiya, and A. N. Lavrov, Phys. Rev. Lett. 88, 137005 (2002).

52 J. M. Tranquada, B. J. Sternlieb, J. D. Axe, Y. Nakamura, and S. Uchida, Nature (London) 375, 561 (1995).

53 J. H. Schon, M. Dorget, F. C. Beuran, X. Z. Zu, E. Arushanov, C. Deville Cavellin, and M. Lagues, Nature (London) 414, 434 (2001).

${ }^{54}$ H. Buttner and A. Blumen, Nature (London) 329, 700 (1987).

${ }^{55}$ M. Prester, Phys. Rev. B 60, 3100 (1999).

${ }^{56}$ S. Capaccioli, M. Lucchesi, P. A. Rolla, and G. Ruggeri, J. Phys.: Condens. Matter 10, 5595 (1998).

${ }^{57}$ M. Giona and H. E. Roman, J. Phys. A 185, 87 (1992). 